\title{
Using Argumentation Strategies in Automated Argument Generation
}

\author{
Ingrid Zukerman, Richard McConachy and Kevin B. Korb \\ School of Computer Science and Software Engineering \\ Monash University \\ Clayton, Victoria 3800, AUSTRALIA \\ email: \{ingrid, ricky, korb\}@csse.monash.edu.au
}

\begin{abstract}
During argumentation, people persuade their audience using a variety of strategies, e.g., hypothetical reasoning, reasoning by cases and ordinary premise-to-goal arguments. In this paper, we offer an operational definition of the conditions for pursuing these strategies, and incorporate into a Bayesian argument-generation system a mechanism for proposing applicable argumentation strategies, generating specific arguments based on these strategies, and selecting a final argument.
\end{abstract}

\section{Introduction}

During argumentation, people persuade their audience using a variety of strategies, e.g., hypothetical reasoning, reasoning by cases and premise to goal. Although the use of different strategies is common in human argumentation, the argumentation and discourse planning systems developed to date offer little insight into the problem of proposing different argumentation strategies and selecting among them.

In this paper, we extend our previous work on argument generation (Zukerman et al., 1998; Zukerman et al., 1999) to address this problem. In this extension, we provide an operational definition of promising conditions for pursuing different argumentation strategies, and incorporate the procedure for selecting an argumentation strategy into the content planning process. The integration of strategy selection and content planning is necessary due to the interplay between argumentation strategy and content: the strategy influences the content that is relevant to an argument, while the information gathered early in the content planning process determines the applicability of the different strategies.

The argumentation strategies discussed in this paper are premise to goal, -hypothetical (reductio ad absurdum and inference to the best explanation) and reasoning by cases (exclusive and non-exclusive) (Figure 1). Premise to goal starts from believed premises and proceeds to the goal. Reductio ad absurdum assumes the negation of the goal, leading to an argument which results in a contradiction with a believed premise and requires the assertion of the

Premise to goal: Corrective lenses are re-
quired.
"Being unable to see far objects is evidence for
myopia, which indicates that corrective lenses
are required."
Reductio ad absurdum: There has always
been matter.
"There could never have been a time when
nothing existed, for, if there were [hypotheti-
cal assumption], then nothing would exist now,
since from nothing comes nothing." (from St.
Thomas Aquinas)
Inference to the best explanation: Patient
has the flu.
"If she had the flu [hypothetical assumption],
she would be tired, achy. and feverish, which
are all true. Hence, she probably has the flu"
Reasoning by cases (exclusive): There can-
not be a utopian society.
"Having checks on procreation leads to misery
and vice, which in turn results in a non-utopian
society.
Having no checks on procreation leads to a pop-
ulation explosion, which in turn leads to star-
vation. This also results in a non-utopian soci-
ety." (from Malthus, 1798 )
Reasoning by cases (non-exclusive): Fail-
ing a test.
"I don't know whether he is stupid or lazy. But
either way, he is likely to fail the test."

Figure 1: Examples of Argumentation Strategies

goal to resolve this contradiction. Inference to the best explanation (Lipton, 1991) assumes the goal, leading to an argument that supports a believed premise (which would be disbelieved in the absence of this assumption), Reasoning by cases enumerates a set of exhaustive conditions and establishes that a desired conclusion would follow regardless of which case is true. ${ }^{1}$ In particular, we consider two types of reasoning-by-cases strategies: exclusive and non-

\footnotetext{
${ }^{1}$ Reasoning by cases is not related to case-based reasoning, which involves problem solving based upon some stereotypical case.
} 
exclusive. The exclusive strategy is applicable to situations where the belief in a proposition is unknown or not agreed upon by the conversational partners. In this case, two separate arguments for the goal are generated, one assuming the truth of this proposition and the other assuming its falsity. ${ }^{2}$ The nonexclusive strategy applies to situations where at least one of several propositions is known to be true, but it is not known which. This strategy produces separate arguments in support of the goal, each of which assumes the truth of one of these propositions.

In the following section, we discuss related research. Next, we present an overview of NAG's argument generation process. We then describe our procedure for proposing argumentation strategies, and for generating and selecting specific arguments. Finally, we illustrate the operation of our mechanism with an example, discuss results from our preliminary evaluation and present concluding remarks.

\section{Related Research}

A general introduction to hypothetical reasoning, including a discussion of counterfactual reasoning and modality, may be found in (Rescher, 1964). The use of suppositions in hypothetical reasoning to create reductio ad absurdum arguments is described in (Freeman, 1991), and their use in the analysis of such arguments is discussed in (Fisher, 1988). Fischer also illustrates how suppositions can lead to arguments that explain observed outcomes, a weaker version of inference to the best explanation. Conditional argumentation, a weaker form of reasoning by cases, where not all the cases must be examined and the beginning of each case does not have to be proven within the argument itself, is described in Freeman's work. These works provide theoretical insights into the field of dialectics. However, they do not present implementable computational mechanisms.

In the area of discourse planning, few systems deal with the selection of argumentation strategies. Cerbah (1992) considers three discourse strategies: CausalChain, which is a special case of our premise to goal strategy; Parallel. which assigns a parallel structure to part of the text; and Concessive. These strategies reflect specific patterns of argumentation which may be incorporated in our higher level strategies. Elhadad (1995) considers the use of argumentative features at several stages of the discourse planning process, but none of his stages deals with high-level argumentation strategies. Reed and Long (1997) use ordering heuristics to model the effect of presentation order on argument persuasiveness, and Marcu (1996) considers the effect of various stylistic factors, including ordering and lexical

\footnotetext{
2The generalization of this strategy to $N$ propositions requires the presentation of $2{ }^{*}$ cases: in the current implementation. only individual propositions are considered.
}

choice, on argument persuasiveness. The mechanisms developed by these researchers, which are applicable after an argumentation strategy has been selected, are expected to complement our future investigation on modeling the effect of rhetorical factors on an addressee's beliefs.

\section{The Argument Generation Process}

The platform for our investigation is the argumentation system NAG (Nice Argument Generator) (Zukerman et al., 1998; Zukerman et. al.;1999). NAG generates nice arguments, that is, arguments that are both normatively correct and persuasive for a target audience. To this effect, it tests the effects of prospective arguments on two models: (1) a normative model, which represents NAG's beliefs, and (2) a user model, which represents a user's presumed beliefs. Each model incorporates a Bayesian network (BN) (Pearl, 1988) as its main representation formalism (BNs were chosen because of their ability to represent normatively correct reasoning under uncertainty). An argument is represented as an Argument Graph, which is a network of nodes that represent propositions, and links that represent the inferences connecting these propositions. This Argument Graph is obtained from the structural intersection of relevant portions of the normative and user BNs. By considering the Argument Graph relative to both models we are able to assess both its normative correctness and its persuasiveness.

NAG receives as input a goal proposition to be argued for, an initial argument context, and a target range for the belief to be achieved in the goal (as a result of the argument) in the user model BN and the normative model BN. Initially, the context is composed of the goal proposition and salient propositions and concepts mentioned in the preceding discussion. During argument generation, the context is expanded to include the current Argument Graph.

Figure 2 shows the main modules of NAG (the modules in double boxes contain the new argumentation strategy mechanisms). After receiving a goal proposition, the Strategist activates a sequence of focusing-generation-analysis cycles as follow's. First, the Attentional Mechanism is invoked to focus on parts of the normative and user BNs that are likely to be useful in the argument. This is performed by spreading activation from the initial context. This process generates an initial Argument Graph, and in later cycles extends the existing Argument Graph. The Strategist then calls the Generator to continue the argument building process by finding additional information to incorporate in the Argument Graph (Zukerman et al., 1998). The extended Argument Graph is returned to the Strategist, which invokes the Analyzer to determine the beliefs in the nodes in the Argument Graph under a variety of condi- 


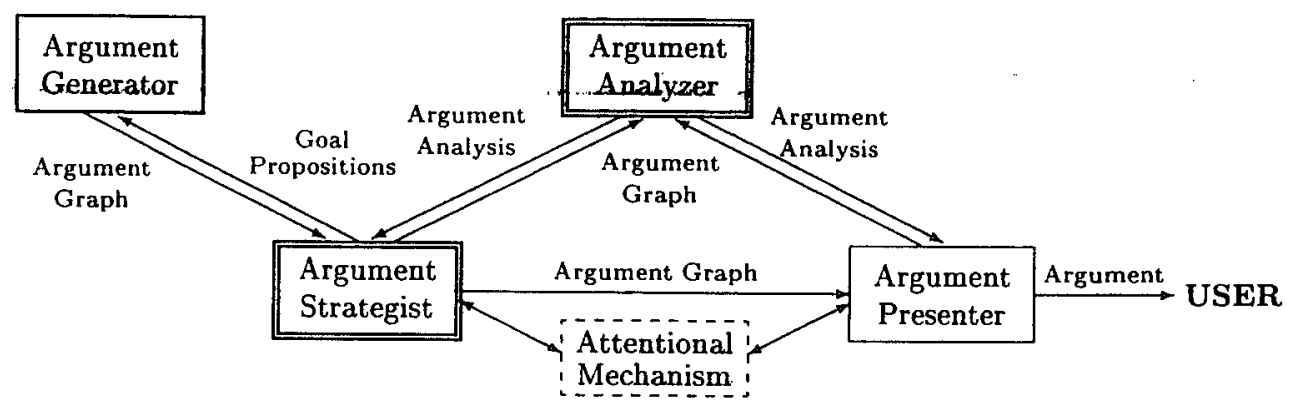

Figure 2: System Architecture

tions (Section 4.1). The Analyzer uses a constrained Bayesian propagation scheme on the normative and user BNs, limiting the updates to the subnetworks represented in the Argument Graph. For the purposes of Bayesian updating, propositions which are provided in the preamble are treated as "observations"; that is, their degrees of belief are used as sources during Bayesian propagation. Based on the beliefs resulting from the Bayesian propagation, the Strategist determines which argumentation strategies are worth pursuing (Sections 4.2 and 4.3). If no strategy yields a nice enough argument, i.e., the belief in the goal is outside the target range in one or both models, the context is expanded, and another generation-analysis cycle is performed: the Strategist re-activates the focusing mechanism, followed by the re-activation of the Generator and then the Analyzer. This process iterates until a successful Argument Graph is built, or NAG is unable to continue, e.g., because it failed to find further evidence.

If one or more strategies yield a nice enough argument, the Strategist selects one of the more concise arguments (Section 4.4). The corresponding Argument Graph and an ordering of the nodes to be presented are then passed to the Presenter, which removes easily inferred propositions from the argument. After each removal, the Presenter activates the Analyzer to check whether the argument remains nice enough, and the Attentional Mechanism to determine whether the argument can still be followed by the user. After the Presenter determines that no more propositions can be removed from the argument, it extracts Bayesian reasoning patterns from the final Argument Graph and passes them to the interface, which renders the argument in English (Zukerman et al., 1999).

This procedure is implemented by the following algorithm, which is executed by the Strategist. ${ }^{3}$

\footnotetext{
${ }^{3}$ A previous version of this procedure which generates only premise-to-goal arguments is described in (Zukerman et al., 1998). In this paper, we focus on Steps 4 and 5, which have been modified to support the consideration of different argumentation strategies during the content planning process.
}

\section{Generation-Analysis Algorithm}

1. Perform spreading activation starting from the items in the current context.

2. Identify new subgoals in the current Argument Graph.

3. Pass the subgoals identified in Step 2 to the Generator, which adds to the current Argument Graph new information related to these subgoals.

4. Pass the Argument Graph generated in Step 3 to the Analyzer for evaluation under different conditions.

5. If (based on the Analyzer's report) some of the argumentation strategies seem promising then

(a) Inspect specific arguments based on these strategies, and

(b) Pass to the Presenter the portion of the Argument Graph corresponding to a concise argument which achieves the intended belief in the goal.

6. Otherwise, add to the current context new nodes that were connected to the goal or became salient during this cycle, and go to Step 1.

\section{Using Argumentation Strategies}

During the argument generation process, the Strategist performs the following actions: (1) determine the potential applicability of the different argumentation strategies based on the beliefs in the nodes in the Argument Graph. (2) propose specific candidates for each applicable strategy, and. (3) select a concise argument among these candidates.

\subsection{Anticipating the effect of a node}

The Strategist selects an argumentation strategy based on the Analyzer's assessment of the effect of the nodes in the Argument Graph on the goal proposition (and vice versa). This effect is determined by means of a constrained Bayesian propagation scheme 
in both the user model $\mathrm{BN}$ and the normative model BN. Specifically, for each node at the "edge" of the Argument Graph, each new node (i.e., one added in the last generation step), and each previous node to which new links were added in the last step, the Analyzer calculates its positive and negative effect on the goal, and the positive and negative effect of the goal on this node. ${ }^{4}$ The positive/negative effect of a node $X$ on a node $Y$ is the hypothetical belief in node $Y$ after propagating a high/low belief in node $X$ (which represents a true/false belief in the corresponding proposition). The positive/negative effect of a node on the goal is required to generate arguments by cases, and the positive/negative effect of the goal on a node is required to generate hypothetical arguments, viz reductio ad absurdum and inference to the best explanation. When computing positive/negative effects for a particular node, the Bayesian propagation process uses the prior beliefs of the other nodes in the Argument Graph.

\subsection{Determining applicable argumentation strategies}

After receiving the Analyzer's report, the Strategist checks the following conditions to determine the potential applicability of each argumentation strategy. ${ }^{5}$

Reductio ad absurdum - The negation of the goal $G$ undermines a proposition $Q$ which is firmly believed independently of the goal (i.e., $P(Q)=$ High, where $Q$ is a premise or inferred from premises). Hence, $P(Q \mid \neg G)=$ Low (where $Q$ is temporarily treated as if it were not a premise, so that its value may change when the goal is negated).

Inference to the best explanation - The assertion of the goal $G$ supports a proposition $Q$ which is firmly believed (i.e., $P(Q)=$ High, where $Q$ is a premise or inferred from premises), but which would be unexplained (improbable) without supposing the truth of the goal. Hence, whereas $P(Q \mid G)=\mathrm{High}$, in the absence of information about $G$, the belief in $Q$ is low (where $Q$ is temporarily treated as if it were not a premise).

Reasoning by cases (exclusive) - A proposition $Q$ satisfies one of the following conditions: (1) it has-an indeterminate level of belief in both the normative and user models (i.e., its probability is within an interval $[0.5 \pm \theta]$ ); or (2) it has highly

\footnotetext{
${ }^{4}$ Previous nodes with new links are reconsidered because their effect on the goal node (and the goal node's effect on them) is more likely to have changed due to these links than the effects of nodes with an unchanged local topology.

${ }^{5}$ For clarity of presentation, these conditions and the subsequent discussion assume a positive bias, i.e., the proposition under consideration is believed: for a negatise bias some expressions will be altered accordingly.
}

divergent levels of belief in the user model and the normative model. . For either condition, the belief in the goal must be high both when a high level of belief is ascribed to $Q$ and when a low level of belief is ascribed.

Reasoning by cases (non-exclusive) - There exists a set of propositions $\left\{Q_{1}, \ldots, Q_{n}\right\}$, each of which leads to a strong belief in the goal (i.e., $P\left(G \mid Q_{i}\right)=$ High for $\left.i=1, \ldots, n\right)$, and the disjunction of these propositions is strongly believed (i.e., $P\left(\bigvee_{i} Q_{i}\right)=$ High). ${ }^{6}$

Premise to goal - This is the default strategy and requires only that given the current beliefs in the premises, the belief in the goal will be in the target range in both the normative and user BNs.

Since the conditions for the reasoning by cases strategies consider nodes in the Argument Graph separately, they do not guarantee that all opportunities to argue by cases will be found. For instance, two particular nodes may not satisfy the conditions for the exclusive strategy when considered separately (because when a node is ascribed a high or low level of belief, the prior beliefs of the other nodes are used for Bayesian propagation). However, when considered jointly, the four permutations of extreme beliefs in these nodes, viz high-high, high-low, low-high and low-low, may satisfy the applicability conditions of the exclusive strategy. At present, these opportunities are missed by NAG. However, this may be an appropriate outcome, since such complex arguments by cases are quite rare.

\subsection{Proposing specific arguments for each strategy}

In this step, the Strategist considers the propositions or sets of propositions that satisfy the conditions for each applicable argumentation strategy, and generates a specific argument based on each of these propositions (or sets of propositions). This is done as follows for each argumentation strategy.

Reductio ad absurdum and Inference to the best explanation. For each proposition $Q$ which satisfies the conditions for reductio ad absurdum, the Strategist extracts from the Argument Graph the subgraph which corresponds to the line of reasoning going from the goal node (which was ascribed a low level of belief) to $Q$ (which has been contradicted

\footnotetext{
${ }^{6}$ This situation may be generalized so that any $Q_{i}$ consists of a subset of propositions which lead to the goal. However, in the current implementation, each $Q_{i}$ consists of one proposition only. Further, owing to practicality considerations, at present NAG implements a limited version of the applicability conditions for the non-exclusive strategy whereby only pairs of nodes that are relatively close to the goal and to observable nodes are inspected. This last requirement is necessary in order to determine which combinations of beliefs are possible for the inspected pairs of nodes.
} 
as a result of this line of reasoning). Each line of reasoning is obtained by treating the negation of the goal as a premise and $\neg Q$ as a goal.

A similar process is applied for the inference to the best explanation strategy, but the goal is ascribed a high level of belief, and $Q$ is expected to achieve a high level of belief as a result of the argument. In general, when using the reductio ad absurdum strategy, people identify only one target proposition to be contradicted when the goal is negated. In contrast, for inference to the best explanation, the goal is often used to explain several propositions. In the current implementation, only one target proposition is being considered for both strategies.

Reasoning by cases (exclusive). If proposition $Q$ satisfies the conditions for the exclusive strategy, then a copy of the Argument Graph is made for the case where a high belief is ascribed to $Q$ and another copy is made for the case where a low belief is ascribed to $Q$. Both copies have the same structure, but the propagated values are different. The argument by cases consists of a pair of Argument Graphs, one graph for each case. These graphs do not require further analysis, since the results of propagating these beliefs through the Argument Graph were previously returned by the Analyzer (Section 4.1), and according to the applicability conditions for the exclusive strategy, the argument for each case is sufficiently nice.

Reasoning by cases (non-exclusive). If a set of nodes $\left\{Q_{1}, \ldots, Q_{n}\right\}$ satisfies the applicability conditions of the non-exclusive strategy, an Argument Graph is generated for each of the $n$ cases by ascribing a high level of belief to each $Q_{i}$ in turn (the rest of the nodes retain their existing degrees of belief). If the Analyzer reports that the argument corresponding to each graph is sufficiently nice, an argument by cases is constructed by listing each graph in turn.

Premise to goal. Finally, the Strategist considers a premise to goal argument by inspecting the belief in the goal in both the normative and user models after propagation from the premises (this belief was computed by the Analyzer). If the argument is nice enough, then it is retained as a possible candidate.

If upon completion of this process, none of these argumentation strategies has yielded a nice enough argument, the reasoning context is updated with nodes that were connected to the goal or became salient during the current cycle. The Strategist then re-invokes the spreading activation process, and reactivates the Generator to expand the Argument Graph (Section 3). After expansion, the analysis and strategy proposal processes are repeated. If one or more candidate arguments were generated, the Strategist selects a concise argument as described in the next section.
When choosing its final argument, the Strategist considers only nice arguments, i.e., those that achieve a degree of belief in the goal which lies inside the target range in both the user model and the normative model. However, we do not have a direct means for determining the belief in the goal in the user model as a result of a hypothetical argument or an argument by cases. This is because the rhetorical force of these strategies affects the user's beliefs in a manner that deviates from the effect modeled by means of Bayesian propagation, as illustrated by the sample arguments in Section 5 . The problem of incorporating a model of the rhetorical force of an argument into a Bayesian propagation scheme is yet to be addressed. Nonetheless, in order to test the operation of our mechanism, we currently approximate the effect of an argument (regardless of its strategy) on the user's beliefs by performing Bayesian propagation in the user model BN. In the future, as a first step in modeling rhetorical factors, we intend to investigate how the beliefs in our user models deviate from users' actual (reported) beliefs.

\subsection{Selecting a concise argument}

Here the Strategist removes long arguments, so that a final selection is made among (shorter) arguments of similar length. ${ }^{7}$ NAG does not simply select the most concise argument, because as shown in Section 6 , the choice of strategy has a greater influence on the addressee's beliefs than any (small) remaining differences in argument length.

The Strategist initially performs coarse pruning on the Argument Graphs that were generated by the premise to goal or reasoning by cases strategies. This coarse-grained pruning examines separately the impact of each individual line of reasoning contributing to the belief in the goal, removing entire lines that are not strictly necessary to achieve a belief in the goal that falls inside the target range (the arguments generated using the reductio ad absurdum and inference to the best explanation strategies are not coarsely pruned, since those arguments already comprise a single line of reasoning). Sometimes, the impact of certain lines of reasoning cannot be assessed in isolation, since two or more lines may contribute jointly towards the belief in a proposition in a mutually dependent manner. Often however, some of the contributing lines of reasoning are independent or nearly so, and coarse pruning can proceed.

Next, the Strategist drops from consideration the arguments that are significantly longer than the shortest argument (where length is measured in number of nodes), ${ }^{8}$ and selects one of the remaining

\footnotetext{
${ }^{7}$ Other factors, such as the structural complexity of the arguments, will be considered in the future.

${ }^{3}$ Although an Argument Graph is further pruned before presenting its corresponding argument to the user (Section 3), it is reasonable to consider the length of each candidate graph
} 


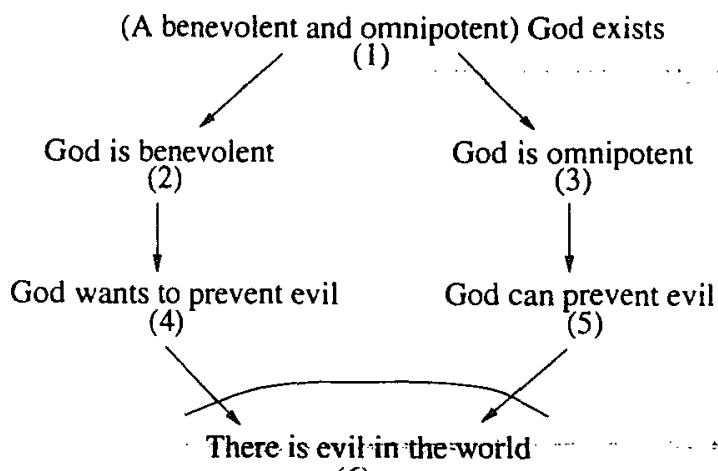

(6)

Figure 3: Argument Graph for the Problem of Evil

arguments according to the following order of preference: reasoning by cases, premise to goal, inference to the best explanation and reductio ad absurdum. This ordering is consistent with the results of our evaluation (Section 6).

\section{Example - The Problem of Evil}

We now illustrate our argumentation mechanism with "The Problem of Evil". Given a preamble that establishes that there is evil in the world, and the goal to prove that there is no God, NAG obtains the Argument Graph in Figure 3 after one focusing-generation cycle, and produces the Argument Graphs corresponding to the arguments in Figure 4 (the adverbs that indicate level of belief and the conjunctive expressions are italicized in the arguments for ease of comparison). ${ }^{9}$ These arguments are based on a definition of God that requires God to be both omnipotent and benevolent.

Premise to goal. Bayesian propagation of the belief in node 6 results in the denial of the combination of nodes 4 and 5 , but yields a moderate probability for each of these nodes and for their respective parents, node 2 and node 3 . Still, the probability of node 1 is quite low (i.e., there is a high belief in its negation).

Reductio ad absurdum. The conditions for reductio ad absurdum are also met by this Argument Graph. That is, the negation of the goal undermines the belief in the premise (the existence of evil).

Reasoning by cases (exclusive). The conditions for exclusive reasoning by cases are met by both node 4 and node 5 , since they obtain middling degrees of belief during propagation. We illustrate here only the argument which hinges on node 4 (the argument which hinges on node 5 is symmetrical). The two cases in the generated argument are:

at this stage, because it is indicative of the length of the argument obtained after finer pruning.

${ }^{9}$ The English versions of these arguments were hand generated from NAG's output. node 4 is true or node 4 is false. The case which assumes the negation of node 4 leads to a.straight: forward argument that achieves the goal. The case which asserts node 4 achieves the goal through an explain away relationship which involves nodes 4,5 and 6 (Pearl, 1988). This relationship requires that $P(6 \mid \neg 4)>P(6)$ and $P(6 \mid \neg 5)>P(6)$, which means that the negation of nodes 4 and 5 are potential explanations for node 6 , and that $P(4 \mid 6 \& 5)<P(4 \mid 6)$ and $P(5 \mid 6 \& 4)<P(5 \mid 6)$, which means that given node 6 , node 5 explains away node 4 and vice versa (Zukerman et al.," 1999)." "That is," asserting "the proposition in node 5 in light of node 6 greatly weakens the belief in node 4 .

Reasoning by cases (non-exclusive). The Strategist identifies nodes 4 and 5 as possible sources for a non-exclusive argument by cases, since the negation of each of these nodes leads to a strong belief in the goal, and $P(\neg 4 \bigvee \neg 5)$ is high (because of their relation to node 6 ). The cases in the generated argument are: node 4 is false or node 5 is false.

Since all these arguments are nice, the Strategist retains all of them for further processing. As stated in Section 4.4, the arguments that are substantially longer than the shortest argument (in number of nodes) are dropped from consideration. In our example, the premise to goal argument is the shortest, as it threads a path through the 6 nodes in the Argument Graph; the exclusive reasoning by cases argument is the longest, requiring 9 nodes ( 3 for the case where node 4 is false, 5 for the case where node 4 is true, and 1 for stating the conclusion); the non-exclusive reasoning by cases argument requires 8 nodes ( 3 for each case, 1 for node 6 , which introduces the cases, and 1 for the conclusion); and the reductio ad absurdum argument requires 7 nodes (the 6 nodes in the Argument Graph plus 1 node for stating the conclusion). The exclusive reasoning by cases argument is dropped from consideration since it is 1.5 times longer than the shortest argument, and the non-exclusive argument is selected among the remaining arguments by applying our preference ordering.

\section{Preliminary Evaluation}

In order to determine the users' preferences for different argumentation strategies, we performed a preliminary evaluation where we presented two sets of arguments to subjects. One set contained the premise to goal and reasoning by cases arguments for the problem of evil shown in Figure 4. The second set contained a preamble which presented some background information, and a premise to goal, a reductio ad absurdum and an inference to the best explanation argument for a large asteroid striking Earth 65 million years BC (Figure 5). The argu- 


\section{Premise to goal:}

"Evil in the world (6) implies that God may not want to prevent evil $(\neg 4)$ and that God may not be able to prevent evil $(\neg 5)$. God possibly not wanting to prevent evil $(\neg 4)$ implies that God may not be benevolent $(\neg 2)$. God possibly not being able to prevent evil $(\neg 5)$ implies that God may not be omnipotent $(\neg 3)$. The fact that God may not be benevolent $(\neg 2)$ and that God may not be omnipotent $(\neg 3)$ implies that it is very likely that God does not exist $(\neg 1)$."

\section{Reductio ad absurdum:}

"Assume that God exists (1). This implies that God is benevolent (2) and that God is omnipotent (3). God being benevolent (2) implies that God wants to prevent evil (4). God being omnipotent (3) implies that God can prevent evil (5). The fact that God wants to prevent evil (4) and that God can prevent evil (5) implies that there is no evil in the world $(\neg 6)$. But there is evil in the world (6): Therefore, Gerd does not exist."

Reasoning by cases (exclusive):

"Consider the following cases: God wants to prevent evil (4), and God does not want to prevent evil $(\neg 4)$.

God wants to prevent evil (4). This, together with the existence of evil (6) implies that God is not able to prevent evil $(\neg 5)$, which in turn implies that God is not omnipotent $(\neg 3)$. This implies that God does not exist $(\neg 1)$.

God does not want to prevent evil $(\neg 4)$. This implies that God is not benevolent $(\neg 2)$, which in turn implies that God does not exist $(\neg 1)$.

Either way, God does not exist $(\neg 1)$."

Reasoning by cases (non-exclusive):

"Since there is evil in the world (6), God does not want to prevent evil $(\neg 4)$ or God cannot prevent evil $(\neg 5)$.

God does not want to prevent evil $(\neg 4)$. This implies that God is not benevolent $(\neg 2)$, which in turn implies that God does not exist $(\neg 1)$.

God cannot prevent evil $(\neg 5)$. This implies that God is not omnipotent $(\neg 3)$, which in turn implies

that God does not exist ( $\neg 1)$.

Either way, God does not exist $(\neg 1)$."

Figure 4: Arguments for the Problem of Evil

ments in each set were presented in two different orders. 40 subjects read the 'problem of evil' arguments, and 35 the 'asteroid' arguments. In the former set, the distribution of preferences was uniform among the three strategies. In the latter set, premise to goal was preferred, followed by inference to the best explanation and then reductio ad absurdum (these results, which were not affected by the order of presentation, were supported by $\chi^{2}$ tests which were significant at the 0.01 level).

At first glance it appears that premise to goal is the preferred argumentation strategy. However, the participants' comments indicate that further experiments are required to determine the conditions under which different argumentation strategies are appropriate. For example, several participants indicated that reductio ad absurdum arguments are appropriate when the ensuing contradiction is compelling, which they did not find to be the case in the asteroid example. Further they stated that they liked the premise to goal argument because it contained more information than the other arguments (which have one line of reasoning only). However. this additional information may be less appealing for arguments that are longer than one paragraph.

\section{Conclusion}

We have offered an operational definition of the conditions for pursuing three types of argumentation strategies: hypothetical, reasoning by cases and premise to goal. We have also presented a mechanism that proposes applicable argumentation strategies based on these conditions, and generates specific arguments based on these strategies. This mechanism has been implemented in a Bayesian argumentgeneration system. Our evaluation also brings to notice the need to investigate additional aspects of argumentation strategies.

\section{Acknowledgments}

This work was supported in part by Australian Research Council grant A49531227.

\section{References}

Cerbah, F. (1992). Generating causal explanations: From qualitative models to natural language texts. In ECAI92 - Proceedings of the. 


\begin{tabular}{l} 
Preamble: \\
"Approximately 65 million years BC the dinosaurs, large reptiles that dominated the Earth for \\
many millions of years, became extinct. At about the same time, the number of giant sequoias in \\
California greatly increased." \\
\hline \hline Premise to goal: \\
"65 million years ago, dinosaurs became extinct and giant sequoias proliferated. These events may \\
have been caused by a cooling of the Earth, which in turn may have been caused by material \\
obstructing the sun. \\
Lots of 65 million year old iridium deposits have been found. This may have been caused by \\
widespread iridium being deposited 65 million years ago, which together with the material ob- \\
structing the sun may have been caused by an explosion which threw up material. This explosion \\
may have been caused by a large iridium-rich asteroid.striking.Earth 65 mallion years. BC."..... \\
\hline Reduction ad absurdum: \\
"If a large iridium-rich asteroid had not struck Earth 65 million years BC, there wouldn't be a \\
large explosion that up throws material. Therefore, iridium would not have spread around, and \\
widespread 65 million year old iridium deposits would not have been found. \\
However, widespread 65 million year old iridium deposits were found. Therefore, a large iridium- \\
rich asteroid struck Earth about 65 million years BC." \\
\hline Inference to the best explanation: \\
"If an iridium-rich asteroid had struck Earth 65 million years BC, there would have been an \\
explosion that throws up material, hence widespread iridium would have been deposited. Therefore, \\
lots of 65 million year old iridium deposits would be found. \\
Since widespread 65 million year old iridium deposits were found, then an iridium-rich asteroid \\
struck Earth 65 million years BC." \\
\hline \hline
\end{tabular}

Figure 5: Arguments for an Asteroid Striking Earth 65 Million Years BC

Tenth European Conference on Artificial Intelligence, pages 490-494, Vienna, Austria.

Elhadad, .M. (1995). Using argumentation in text generation. Journal of Pragmatics, 24:189-200.

Fisher, A. (1988). The logic of real arguments. Cambridge University Press, Cambridge: England.

Freeman, J. (1991). Dialectics and the macrostructure of arguments: a theory of argument structure. Foris Publications, Berlin.

Lipton, P. (1991). Inference to the best explanation. Routledge, London; New York.

Malthus, T. (1798). Essay on the Principle of Population as it affects the Future Improvement of Society with Remarks on the Speculations of Mr. Godwin, Mr. Condorcet and other Writers.

Marcu, D. (1996). The conceptual and linguistic facets of persuasive arguments. In Proceedings of ECAI-96 Workshop - Gaps and Bridges: New Directions in Planning and $N L G$, pages $43-46$, Budapest, Hungary.

Pearl, J. (1988). Prababilistic Reasoning in Intelligent Systems. Morgan Kaufmann Publishers, San Mateo, California.

Reed. C. and Long, D. (1997). Content ordering in the generation of persuasive discourse. In $I J$ CA197 - Proceedings of the Fifteenth International Joint Conference on Artificial Intelligence. pages 1022-102i, Nagoya, Japan.
Rescher, N. (1964). Hypothetical reasoning. North Holland, Amsterdam.

Zukerman, I., McConachy, R., and Korb, K. B. (1998). Bayesian reasoning in an abductive mechanism for argument generation and analysis. In AAAI98 - Proceedings of the Fifteenth National Conference on Artificial Intelligence, pages 833838, Madison, Wisconsin.

Zukerman, I., McConachy, R., Korb, K. B., and Pickett, D. A. (1999). Exploratory interaction with a Bayesian argumentation system. In $I J$ CAI99 - Proceedings of the Sixteenth International Joint Conference on Artificial Intelligence, pages 1294-1299, Stockholm, Sweden. 